\title{
ON COALGEBRAS AND TYPE TRANSFORMATIONS
}

\author{
H. PETER GUMM
}

\begin{abstract}
We show that for an arbitrary Set-endofunctor $T$ the generalized membership function given by a sub-cartesian transformation $\mu$ from $T$ to the filter functor $\mathbb{F}$ can be alternatively defined by the collection of subcoalgebras of constant $T$-coalgebras. Sub-natural transformations $\varepsilon$ between any two functors $S$ and $T$ are shown to be sub-cartesian if and only if they respect $\mu$. The class of $T$-coalgebras whose structure map factors through $\varepsilon$ is shown to be a covariety if $\varepsilon$ is a natural and sub-cartesian mono-transformation.
\end{abstract}

\section{SET-FunCtORS}

Our interest in $\mathcal{S}$ et-Functors arises from their use as signatures of algebras or coalgebras. A Set-functor $T$ associates with each set $X$ a set $T(X)$ and with each map $f: X \rightarrow Y$ between sets a map $T f: T(X) \rightarrow T(Y)$ so that identities and function compositions are preserved, i.e. $T i d_{X}=i d_{T(X)}$ and $T(g \circ f)=T g \circ T f$ whenever $f: X \rightarrow Y$ and $g: Y \rightarrow Z$.

In the context of universal algebra, the most important examples are given by the so called polynomial functors. Starting with a sequence of natural numbers $\Delta=\left(n_{i}\right)_{i \in I}$ (called a similarity type) define $T_{\Delta}(X):=\uplus_{i \in I} X^{n_{i}}$ for each set $X$ and put $(T f)\left(x_{1}, \ldots, x_{n_{i}}\right)=\left(f\left(x_{1}\right), \ldots, f\left(x_{n_{i}}\right)\right)$ for each $\left(x_{1}, \ldots, x_{n_{i}}\right) \in X^{n_{i}}$. Then a universal algebra of signature $\Delta$ on a set $A$ is just a map $f^{A}: T(A) \rightarrow A$, and a homomorphism to another algebra $f^{B}: T(B) \rightarrow B$ is just a map $\varphi: A \rightarrow B$ with $\varphi \circ f^{A}=T \varphi \circ f^{B}$.

1.1. Coalgebraic type functors. In the dual context of coalgebras a wide range of functors is of interest in order to model automata, transition systems, probabilistic and nondeterministic systems, processes or even topological spaces. Much of the (co)algebraic theory hinges on particular preservation properties of the $\mathcal{S}$ et-functors defining their signature. Examples of functors, acting on a set $X$ and an arbitrary map $f: X \rightarrow Y$ are:

- the power set functor $\mathbb{P}$, where $\mathbb{P}(X)$ is the power set of $X$ and $(\mathbb{P} f)(U):=$ $f[U]:=\{f(u) \mid u \in U\}$,

- the filter functor $\mathbb{F}$ with $\mathbb{F}(X)$ the set of all filters on $X$ and $(\mathbb{F} f)(\mathcal{G}):=$ $\left\{V \subseteq Y \mid f^{-1}(V) \in \mathcal{G}\right\}$,

- the distribution functor, where $\mathcal{D}(X)$ is the set of all probability distributions on $X$ and $(\mathcal{D} f)(\sigma)(y)=\sum_{f(x)=y} \sigma(x)$,

- the automaton functor $\mathcal{A}(X)=D \times X^{E}$ where $E$ is a fixed input set, $D$ a fixed output set and $X^{E}$ the set of all maps from $E$ to $X$,

- the stream functor $(-)^{\infty}$, where $X^{\infty}$ is the set of all infinite lists (streams) of elements of $X$,

- the binary-tree-functor where $\operatorname{BinTree}(X)$ is the set of all binary trees with leafs from $X$, or, more general 
- the free-algebra-functor for a variety $\mathfrak{V}$, where $\mathcal{F}_{\mathfrak{V}}(X)$ is the free $\mathfrak{V}$-algebra over $X$ and $\mathcal{F}_{\mathfrak{V}} f$ is the homomorphic extension of $f$.

Subfunctors of some of the above functors are of interest, too, such as e.g.

- the list functor $(-)^{\star}$ where $X^{\star}$ is the set of all finite lists (words) of elements of $X$,

- the bounded powerset functors $\mathbb{P}_{\kappa}$ for any cardinal $\kappa$, yielding only the subsets of cardinality below $\kappa$,

- the $(-)_{2}^{3}$-functor, see [1], where $(X)_{2}^{3}:=\left\{\left(x_{1}, x_{2}, x_{3}\right) \in X^{3}|| x_{1}, x_{2}, x_{3} \mid \leq\right.$ $2\}$ and maps are extended componentwise.

Regarding the coalgebraic theories in which these functors occur, certain preservation properties play a fundamental role. In particular, much of the early literature in coalgebra assumed that the type functor $T$ should preserve weak pullbacks, see [9]. Here a weak pullback is a weak limit of two arrows with common target. It was then shown in [7] that a $\mathcal{S}$ et-functor $T$ weakly preserves pullbacks iff it weakly preserves kernels and preimages. (Recall that a kernel is the pullback of an arrow with itself and a preimage, also known as inverse image, is a pullback along a mono, see [2].)

1.2. Container functors and their membership. In computer science, typical functors arise as container datatypes (arrays, lists, trees, streams), where $T(X)$ can be interpreted as the set of all containers of a certain type with elements from $X$. Given an item $u$ in $T(X)$, it is legitimate to ask for the set of all elements that are present in the container represented by $u \in T(X)$. A natural way to formalize this would be to ask for the smallest $U \subseteq X$ such that $u \in T(U)$. But one encounters two difficulties:

- Firstly, $T(U)$ need not be a subset of $T(X)$ even when $U \subseteq X$, unless the functor is standard. However, we can replace $T(U)$ by $\left(T \subseteq \subseteq_{U}^{X}\right)[T(U)]$ which is the image of $T(U)$ under the $T$-image of the inclusion map $\subseteq_{U}^{X}$. Now $\left(T \subseteq_{U}^{X}\right)[T(U)]$ is a subset of $T(X)$ and it is equal to $T(U)$, whenever $T$ is standard. The filter functor $\mathbb{F}$ provides an example of a functor, which is not standard, and preserves weak pullbacks, but not infinite intersections, see [4].

- Secondly, a smallest $U$ with the required properties need not exist. Thus the question for arbitrary elements $u \in T(X)$ need not have a unique answer, but rather a collection of possible answers. Fortunately, this collection is closed under supersets and finite intersections, i.e. it is a filter.

To be precise, the following was defined in [5] for an arbitrary $\mathcal{S} e$-functor $T$ and an arbitrary element $u \in T(X)$ :

$$
\mu_{X}^{T}(u):=\left\{U \subseteq X \mid u \in\left(T \subseteq_{U}^{X}\right)[T(U)]\right\}
$$

and it was shown that $\mu^{T}$, for an arbitrary $\mathcal{S}$ et-functor $T$, is a transformation to the filter functor. $\mu^{T}$ is not a natural transformation in general, but it is always sub-natural, even sub-cartesian, terms which are defined below. In fact, it was proved there :

Theorem 1.1. For any Set-endofunctor $T$

- $\mu^{T}$ is the largest sub-cartesian transformation from $T$ to the filter functor,

- $\mu^{T}$ is natural if and only if $T$ (weakly) preserves preimages. 
Here, a transformation $\sigma$ between two functors $F$ and $G$ is just a collection of maps $\sigma_{X}: F(X) \rightarrow G(X)$ for each set $X$. The transformation is natural, if for each map $f: X \rightarrow Y$ the following diagram commutes:

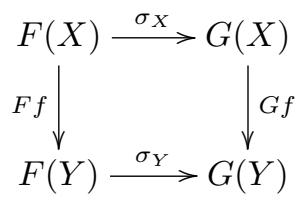

$\sigma$ is called sub-natural, when this square is required to commute only for $f$ injective and is called sub-cartesian, if for each injective $f$ the above diagram is a preimage diagram, i.e. a pullback.

In the above sense then, $\mu$ provides a generalized membership relation for arbitrary functors $T$, assigning to each container $u \in T(X)$ its filter of elements.

The straightforward definition $\sigma_{X}^{T}(u):=\bigcap \mu_{X}^{T}(u)$ always yields the largest subnatural transformation from $T$ to the powerset functor $\mathbb{P}$. If there exists any subcartesian transformation at all from $T$ to $\mathbb{P}$, then $\sigma$ itself is sub-cartesian, and this is the case if and only if $T$ preserves intersections, see [5].

1.3. Sub-cartesian means preservation of membership. We shall provide a criterion for determining, when a general sub-natural transformation $\varepsilon$ between any two functors is actually sub-cartesian. For its proof, we shall require the following easy lemma, see e.g. [8]:

Lemma 1.2. In any category, suppose that the following digram commutes.

(1) If $(A)$ and $(B)$ are pullbacks, then so is the perimeter $(A, B)$.

(2) If $(A, B)$ is a pullback and $f, g$ are jointly monic, then $(A)$ is a pullback.

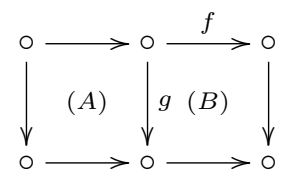

With that we can prove the following characterization:

Theorem 1.3. Let $S$ and $T$ be Set-endofunctors. A sub-natural transformation $\varepsilon$ : $S \rightarrow T$ is sub-cartesian, if and only if it commutes with the generalized membership $\mu$ :

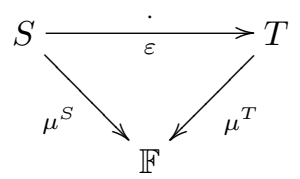

Proof. Let $\varepsilon$ be sub-natural, then for any $U \subseteq X$ the following diagram commutes.

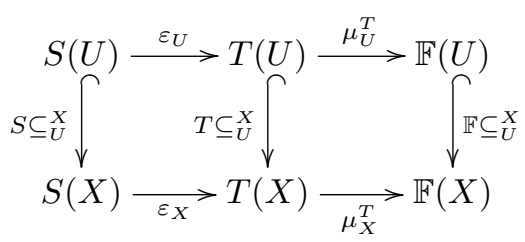


By assumption, $\mu_{U}^{T} \circ \varepsilon_{U}=\mu_{U}^{S}$ and $\mu_{X}^{T} \circ \varepsilon_{X}=\mu_{X}^{S}$, so by theorem 1.1, the perimeter of the diagram is a pullback. Lemma 1.2, therefore guarantees that the left square is a pullback, too, which means that $\varepsilon$ is sub-cartesian.

For the converse assume that $\varepsilon$ is sub-cartesian., we need to show $\mu^{S}=\mu^{T} \circ \varepsilon$. From the first assertion of lemma 1.2 one obtains that $\mu^{T} \circ \varepsilon$ is a sub-cartesian transformation from $S$ to the filter functor $\mathbb{F}$. Therefore, from the first item of theorem 1.1, we obtain the inclusion $\mu_{X}^{T}\left(\varepsilon_{X}(u)\right) \subseteq \mu_{X}^{S}(u)$.

For the reverse inclusion, let any $U \in \mu_{X}^{S}(u)$ be given, then by definition of $\mu$ there exists $v \in S(U)$ with $\left(S \subseteq_{U}^{X}\right)(v)=u$. It follows that $\varepsilon_{X}(u)=\varepsilon_{X} \circ\left(S \subseteq_{U}^{X}\right.$ )$(v)=\left(T \subseteq_{U}^{X}\right) \circ \varepsilon_{U}(v) \in\left(T \subseteq_{U}^{X}\right)[T(U)]$, hence $U \in \mu_{X}^{T}\left(\varepsilon_{X}(u)\right)$.

\section{Conlgebras}

An important property true in the category of sets is that every epi-mono-square has a (necessarily unique) diagonal. That is, given a square $m \circ f=g \circ e$, where $e$ is epi and $m$ mono, there is a unique $d$ such that $d \circ e=f$ and $m \circ d=g$.

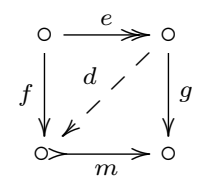

Let $T: \mathcal{S}$ et $\rightarrow \mathcal{S}$ et be any functor. By a $T$-coalgebra we understand a pair $\mathcal{A}=\left(A, \alpha_{\mathcal{A}}\right)$ consisting of a set $A$ and a map $\alpha_{\mathcal{A}}: A \rightarrow T(A)$. A homomorphism $\varphi$ to another $T$-coalgebra $\mathcal{B}=\left(B, \alpha_{\mathcal{B}}\right)$ is just a map making the obvious diagram commute:

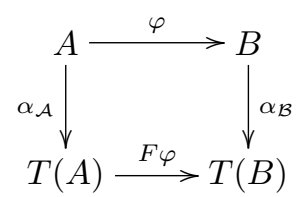

The class of all $T$-coalgebras with homomorphisms as defined above forms a category $\mathcal{S e t}_{T}$, in which all colimits exist. In fact, the forgetful functor, associating with a coalgebra $\mathcal{A}=\left(A, \alpha_{\mathcal{A}}\right)$ its base set $A$, creates and reflects colimits. Since in any category a morphism $\varphi: A \rightarrow B$ is epi if and only if the pushout with itself is the identity $i d_{B}$, this implies that $\varphi$ is epi in $\mathcal{S e t}_{T}$ if and only if it is epi in $\mathcal{S} e t$, i.e. surjective, see [9]. Monos in $\mathcal{S e t}_{T}$, however, need not be injective. Rather, they are injective iff they are regular in $\mathcal{S e t}_{T}$, see [7].

Given a homomorphism $\varphi$ as above, the image factorization in $\mathcal{S e t}$ of $\varphi$ as $\varphi=$ $\subseteq \circ \varphi^{\prime}$ yields an image factorization of $\varphi$ in $\mathcal{S}_{t}$. This is because any $\mathcal{S} e$-functor $\bar{T}$ preserves (nonempty) monos, so the factorization of $\varphi$ in $\mathcal{S}$ et becomes an epimono-square. Now, the structure map on the image $\varphi[A]$ of $A$ under $\varphi$ is provided by the unique diagonal:

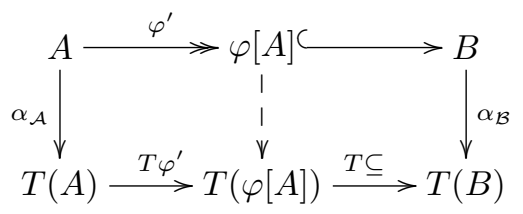


A coalgebra $\mathcal{U}=\left(U, \alpha_{\mathcal{U}}\right)$ is called subcoalgebra of $\mathcal{A}=\left(A, \alpha_{\mathcal{A}}\right)$, provided that $U \subseteq A$ and the structure map $\alpha_{\mathcal{U}}$ is the restriction of $\alpha_{\mathcal{A}}$ in the sense that the embedding $\subseteq_{U}^{A}$ is a homomorphism:

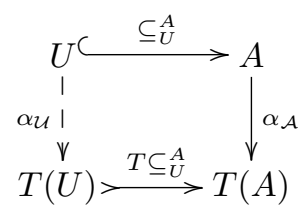

Not every subset $U$ of $A$ supports a subcoalgebra structure, but if it does, the structure map $\alpha_{\mathcal{U}}$ is uniquely determined. By abuse of notation, we therefore shall call such a subset $U$ a subcoalgebra of $\mathcal{A}$.

Finally, the sum $\sum_{i \in I} \mathcal{A}_{i}$ of a family $\left(\mathcal{A}_{i}\right)_{i \in I}$ of coalgebras has as carrier set the sum (in $\mathcal{S} e t$ ) of the carriers of the $\mathcal{A}_{i}$, which is the disjoint union $\uplus_{i \in I} A_{i}$ and as structure map the disjoint union of the $\alpha_{\mathcal{A}_{i}}$.

Given a class $\mathfrak{K}$ of coalgebras, we denote by $S(\mathfrak{K}), H(\mathfrak{K})$, resp. $\Sigma(\mathfrak{K})$ the classes of all subcoalgebras, homomorphic images, resp. sums, of members from $\mathfrak{K}$. A class of $T$-coalgebras closed under the operators $S, H$, and $\Sigma$ is called a covariety, and it is well known that for any class $\mathfrak{K}$ of coalgebras, the smallest covariety containing $\mathfrak{K}$ is given by $S H \Sigma(\mathfrak{K})$ (see for instance[3], where a Birkhoff-Theorem for covarieties is proved).

2.1. Membership through constant coalgebras. We now show that the membership transformation $\mu: T \rightarrow \mathbb{F}$ has an interpretation in coalgebras. For an arbitrary set $X$ and an arbitrary element $c \in T(X)$, let $\kappa^{c}: X \rightarrow T(X)$ be the map with constant value $c$ and let $\mathcal{K}_{X}^{c}=\left(X, \kappa^{c}\right)$ be the constant coalgebra on $X$ with value $c$. Let $\operatorname{Sub}\left(\mathcal{K}_{X}^{c}\right)$ be the collection of subcoalgebras of $\mathcal{K}_{X}^{c}$, then we have:

Proposition 2.1. $\mu_{X}(c)=S u b\left(\mathcal{K}_{X}^{c}\right)$.

Proof. For $U \subseteq X$ we have:

$$
\begin{aligned}
U \leq \mathcal{K}_{X}^{c} & \Longleftrightarrow \exists \alpha: U \rightarrow T(U) \cdot(U, \alpha) \leq \mathcal{K}_{X}^{c} \\
& \Longleftrightarrow \exists \alpha: U \rightarrow T(U) \cdot\left(T \subseteq_{U}^{X}\right) \circ \alpha=\kappa_{c} \circ \subseteq_{U}^{X} \\
& \Longleftrightarrow \kappa^{c}[U] \subseteq\left(T \subseteq_{U}^{X}\right)[T U] \\
& \Longleftrightarrow c \in\left(T \subseteq_{U}^{X}\right)[T(U)] \\
& \Longleftrightarrow U \in \mu_{X}(c) .
\end{aligned}
$$

The collection of all subcoalgebras of a fixed coalgebra is known to be closed under finite intersections, see [6]. In the case of constant coalgebras, it is immediately checked that supersets of subcoalgebras are subcoalgebras, hence this proposition immediately shows that $\mu$ is indeed a transformation to the filter functor.

2.2. $\varepsilon$-crisp coalgebras. In [10], Smith defines a $Q$-iterated function system $(Q$ IFS) as a $Q$-indexed family of stochastic linear maps on a vector space $\mathbb{R}(X)$. Since each linear map is determined by its restriction as $\mathcal{S e t}$-map $\alpha: X \rightarrow \mathbb{R}(X)$, a stochastic linear map is given by any mapping from $X$ to the set of probability 
distributions on $X$, that is as a coalgebra of type $\mathcal{D}(X)$. A $Q$-IFS is therefore a $Q$ indexed family of probabilistic transition systems, that is any map $\rho: Q \rightarrow \mathcal{D}(X)^{X}$. Equivalently, $\rho$ can be encoded in a map $\alpha: X \rightarrow \mathcal{D}(X)^{Q}$, that is a single coalgebra of the $Q$-th power of the distribution functor.

Smith calls such an $\alpha$ crisp, if for each $q \in Q$ there is some $y \in X$ with $\alpha(x)(q)=$ $\hat{y}(x)$, where

$$
\hat{y}(x):= \begin{cases}1 & \text { if } x=y \\ 0 & \text { otherwise }\end{cases}
$$

so $\hat{y}$ is the trivial distribution giving $y$ probability 1 and everything else probability 0 . He then proves that homomorphic images of crisp coalgebras are crisp.

Here we show that the concept is meaningful for arbitrary functors $T$ serving as types of coalgebras, and that in a situation as above, crisp coalgebras even form a covariety. The key is observing that $y \mapsto \hat{y}$ is a transformation between the $(Q$-th powers of) the identity functor $I$ and the functor $\mathcal{D}$ whose properties determine the closure properties of the class of all crisp coalgebras. Thus, we shall define crispness with respect to a fixed transformation $\varepsilon$ from some functor $S$ to $T$.

Definition 2.2. Let $S$ and $T$ be set-endofunctors and let $\varepsilon: S \rightarrow T$ be a transformation, i.e. a collection of maps $\varepsilon_{X}: S(X) \rightarrow T(X)$, one for each set $X$. A $T$-coalgebra $\mathcal{A}=\left(A, \alpha_{\mathcal{A}}\right)$ will be called $\varepsilon$-crisp, provided its structure map factors through $\varepsilon_{A}$. The structure of the class of all $\varepsilon$-crisp coalgebras then depends on the properties of $\varepsilon$ :

Theorem 2.3. Let $\varepsilon: S \rightarrow T$ be a natural and subcartesian mono-transformation. Then the class of all $\varepsilon$-crisp coalgebras forms a covariety.

In fact, we shall give conditions on $\varepsilon$ so that the class of $\varepsilon$-crisp coalgebras are closed under sums, homomorphic images and subcoalgebras. The theorem therefore follows from the following lemma:

Lemma 2.4. Let $\varepsilon: S \rightarrow T$ be a mono-transformation, and let $\mathfrak{K}_{\varepsilon}$ be the class of all $\varepsilon$-crisp coalgebras.

(1) $\mathfrak{K}_{\varepsilon}$ is closed under sums, if $\varepsilon$ is subnatural.

(2) $\mathfrak{K}_{\varepsilon}$ is closed under homomorphic images, if $\varepsilon$ is natural and mono.

(3) $\mathfrak{K}_{\varepsilon}$ is closed under subcoalgebras, if $\varepsilon$ is sub-cartesian.

Proof. Let $\mathcal{A}=\left(\sum_{i \in I} A_{i}, \alpha\right)$ be the sum of the coalgebras $\mathcal{A}_{i}=\left(A_{i}, \alpha_{i}\right)_{i \in I}$ with the sum embeddings $e_{i}: \mathcal{A}_{i} \rightarrow \mathcal{A}$. If all $\mathcal{A}_{i}$ are $\varepsilon$-crisp, we have for each $i \in I$ the following diagram, where the perimeter commutes, since the $e_{i}$ are homomorphisms and the lower square commutes since the $e_{i}$ are injective and $\varepsilon$ is assumed subnatural. We need to construct the indicated map $\sigma$.

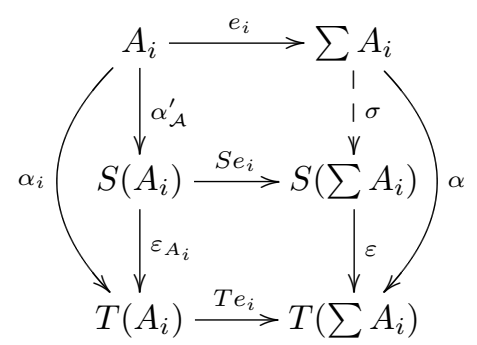


Since $\sum_{i \in I} A_{i}$ with the embeddings $e_{i}$ is the sum of the sets $A_{i}$ in the category Set, we obtain $\sigma$ as the unique map making the upper rectangle commute for each $i \in I$. A diagram chase yields $\varepsilon_{\Sigma} \circ \sigma \circ e_{i}=\alpha \circ e_{i}$ for each $i$, from which $\varepsilon_{\Sigma} \circ \sigma=\alpha$ follows, as the sum embeddings are jointly epi.

Given an $\varepsilon$-crisp $T$-coalgebra $\mathcal{A}=\left(A, \alpha_{\mathcal{A}}\right)$ and an epimorphism $\varphi$ onto a second $T$-coalgebra $\mathcal{B}=\left(B, \alpha_{\mathcal{B}}\right)$, we obtain the diagram.

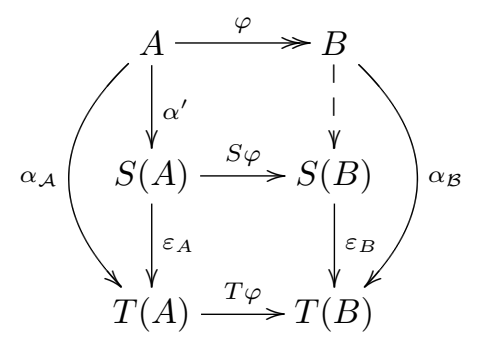

Again, the perimeter commutes, since $\varphi$ is a homomorphism, and the lower square does, since we now assume $\varepsilon$ to be natural. Notice that $\alpha_{\mathcal{B}} \circ \varphi=\varepsilon_{B} \circ S \varphi \circ \alpha^{\prime}$ delineates an epi-mono-square, so $\sigma$ can be obtained as its unique diagonal.

Finally, assume that $\mathcal{A}=\left(A, \alpha_{\mathcal{A}}\right)$ is a subcoalgebra of the $\varepsilon$-crisp coalgebra $\mathcal{B}=\left(B, \alpha_{\mathcal{B}}\right)$. In the diagram below, the lower square is a preimage, and $A$ with $\alpha_{\mathcal{A}}$ and $\alpha_{\mathcal{B}}^{\prime} \circ \subseteq_{A}^{B}$ presents a competitor to this limit, thus yielding the required structure map $\sigma: A \rightarrow S(A)$.

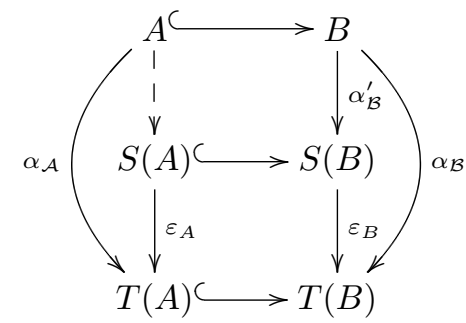

Now the case of $[10]$ is captured easily, as $\varepsilon_{X}: I^{Q} \rightarrow \mathcal{D}^{\mathcal{Q}}$ where $\varepsilon_{X}(\tau)(q)=\widehat{\tau(q)}$, which inherits from $x \mapsto \hat{x}$ the property of being mono, natural, and sub-cartesian.

\section{REFERENCES}

[1] P. Aczel and N. Mendler. A final coalgebra theorem. In D.H. Pitt et al., editors, Proceedings category theory and computer science, Lecture Notes in Computer Science, pages 357-365. Springer, 1989.

[2] S. Awodey. Category Theory. Oxford University Press, 2006.

[3] H.P. Gumm. Birkhoff's variety theorem for coalgebras. erscheint in Contributions to General Algebra, 2000

[4] H.P. Gumm. Functors for coalgebras. Algebra Universalis, 45:135-147, 2001.

[5] H.P. Gumm. From T-coalgebras to filter structures and transition systems. In D.H. Fiadeiro et al., editors, Algebra and Coalgebra in Computer Science, volume 3629 of Lecture Notes in Computer Science, pages 194-212. Springer, 2005.

[6] H.P. Gumm and T. Schröder. Coalgebras of bounded type. Math. Struct. in Comp. Science, 12:565-578, 2002.

[7] H.P. Gumm and T. Schröder. Types and coalgebraic structure. Algebra Universalis, 53:229$252,2005$.

[8] E.G. Manes. Implementing collection classes with monads. Math. Struct. in Comp. Science, 8:231-276, 1998. 
[9] J.J.M.M. Rutten. Universal coalgebra: a theory of systems. Theoretical Computer Science, (249):3-80, 2000.

[10] J.D.H. Smith. Permutation representations of left quasigroups. Algebra Universalis, to appear. 